\title{
Lire, comprendre, interpréter, sans expliquer
}

\section{Dominique Bucheton}

\section{(2) OpenEdition}

Journals

Édition électronique

URL : http://journals.openedition.org/trema/1586

DOI : 10.4000/trema.1586

ISSN : 2107-0997

\section{Éditeur}

Faculté d'Éducation de l'université de Montpellier

\section{Édition imprimée}

Date de publication : 1 octobre 2002

Pagination : 67-76

ISSN : 1167-315X

\section{Référence électronique}

Dominique Bucheton, «Lire, comprendre, interpréter, sans expliquer », Tréma [En ligne], 19 | 2002, mis en ligne le 01 octobre 2002, consulté le 10 décembre 2020. URL : http://journals.openedition.org/ trema/1586 ; DOI : https://doi.org/10.4000/trema.1586

Ce document a été généré automatiquement le 10 décembre 2020.

Trema 


\title{
Lire, comprendre, interpréter, sans expliquer
}

\author{
Dominique Bucheton
}

\section{Introduction}

1 On ne peut aborder la question du : « lire - comprendre - interprêter - doter les élèves de beaucoup de culture » (résumé abrupte, certes!) que les nouvelles I.O. préconisent, sans essayer de réfléchir aux problèmes didactiques et pédagogiques nouveaux qu'elles posent aux enseignants.

2 La dernière mention :" pas d'explications de textes », soulignée avec insistance dans les I.O. comme dans les propos oraux des diverses instances de l'Inspection générale ${ }^{1}$ appelle à une véritable révolution pédagogique des pratiques (plus de questions fermées de compréhension pour l'étude d'un texte!). Quant au couple compréhension interprétation, les recherches théoriques diverses sur la question ${ }^{2}$ ont montré les tensions, entrelacs et cheminements divers, que ces deux concepts entretenaient. Elles ont montré aussi le rôle intro-projectif (Jorro, 1994) ${ }^{3}$ que le langage jouait pour extérioriser des processus de compréhension forcément privés et internes. Il reste aux didacticiens à cerner lesquelles de ces tensions, imbrications de stratégies diverses pour la construction du sens, à des niveaux de signification feuilletés, sont pertinentes et transposables pour de jeunes élèves de l'élémentaire, quelles postures d'enseignement nouvelles, quels gestes professionnels sont nécessaires pour libérer cet espace interprétatif préconisé pour les élèves. Aux didacticiens donc de poursuivre le travail de théorisation didactique de l'enseignement de la littérature pour le premier degré : trier les contenus d'enseignement vraiment fondateurs ${ }^{4}$, élaborer des principes didactiques, repérer les tâches et situations particulièrement favorables au cheminement interprétatif des élèves, travailler des exemples de séquences, bref, élaborer une didactique de la littérature à l'école primaire visant tout à la fois le développement des compétences de lecture littéraire attendues et le développement singulier, psychologique, culturel et social de l'élève. 
3 On peut imaginer que les modèles didactiques de la lecture littéraire à l'instar des modélisations didactiques diverses de l'enseignement de l'écriture, seront multiples et permettront de couvrir de manière large l'ensemble des problèmes que pose l'entrée en littérature préconisée. À l'heure actuelle, deux équipes de recherche $\mathrm{e}^{5}$ ont publié des travaux didactiques conséquents, proposé des pistes de travail concrètes pour l'école élémentaire. Il reste beaucoup à faire pour expliquer l'appropriation diversifiée par les élèves des savoirs, valeurs, contenus culturels véhiculés par les textes littéraires. Des renversements pédagogiques importants sont à opérer pour donner un véritable statut dans la classe au lecteur singulier ${ }^{6}$.

\section{Les nouvelles I.O. : entre avancée saluée et difficultés prévisibles}

4 Les nouvelles I.O. Françaises pour l'école élémentaire donnent à la littérature un statut nouveau (voir ici même Chabanne). Elles introduisent des ruptures importantes dans les conceptions et pratiques pédagogiques de l'enseignement de la lecture littéraire. Elles posent aux jeunes enseignants débutants, par le flou théorique caractéristique de ce genre d'écrit officiel, une série de questions nouvelles, fort stimulantes. Pendant leur première année de formation, ils ont rencontré dans les manuels et leur guide d'accompagnement, dans les discours professionnels des enseignants-formateurs, toute une série de concepts en usage dans le milieu professionnel: "lecture-déchiffrage ", " compréhension-globale ", " compréhension-fine », accompagnés chacun d'une batterie de tâches et questionnements bien identifiables. Aujourd'hui, dans ce bel équilibre, arrive avec les nouvelles I.O., le concept d'interprétation. Qu'en faire? Introduit-il oui ou non des changements dans les conceptions officielles de la lecture? Permet-il de définir la lecture littéraire, si oui à quel niveau? Que va-t-il falloir modifier dans les pratiques pédagogiques ordinaires?

5 Mais surtout quels savoirs nouveaux, quelles pratiques faudra-t-il introduire en formation pour préparer les enseignants à ce nouveau challenge : accompagner les élèves, dans leur diversité sociale et culturelle à devenir les lecteurs avertis, intéressés, enthousiastes d'une littérature très féconde, écrite pour eux.

6 Pour témoigner des questions et difficultés telles qu'elles se posent sur le terrain de la formation, je citerai en introduction des extraits de deux mémoires professionnels soutenus en mai 2002. Ils explorent la question de la lecture interprétative et se livrent à une lecture critique et comparée des I.O. de 1995 et de celles de $2002^{7}$. Ils observent comment la question de la littérature fait son entrée officielle dans les programmes du primaire en se distinguant de la lecture et comment le concept d'interprétation vient semer le trouble dans un édifice didactique jusqu'alors bien balisé.

7 «Dans les I.O. de 1995 », écrit Virginie Signoret, à la fin du cycle III "l'élève doit avoir acquis une bonne maitrise de la lecture, c'est-à-dire être capable de lire silencieusement aussi bien que de lire à voix haute et de façon expressive " ${ }^{8}$. Parmi ces compétences on peut remarquer un travail axé sur la compréhension, l'expression et le déchiffrage. Déchiffrer et comprendre semblent être les deux compétences majeures à acquérir à l'école élémentaire. Aucune remarque n'est faite concernant un quelconque travail d'interprétation de la littérature. L'enfant doit juste« savoir présenter un avis personnel et argumenté sur ce qui a été lu» [...]. Le concept de compréhension fine est défini par les auteurs de "maitrise de la langue » 
comme «la capacité à suppléer au manque du texte, à déduire des informations explicites d'autres informations qui en découlent nécessairement». La compréhension fine, ajoute la stagiaire, serait alors une compréhension de l'implicite présent dans le texte. La compréhension fine paraît être un second degré de la compréhension.

Examinant ensuite les nouvelles compétences attendues, elle ajoute : Dans les nouveaux programmes (2002), les compétences devant être acquises en fin de cycle III ont largement évolué [...] L'enseignant devra rendre les élèves autonomes face à la littérature de jeunesse. L'élève doit la comprendre et en proposer une interprétation sans l'aide de l'adulte. "Le texte des I.O. nouvelles est alors largement cité : Ces rencontres avec les œuvres passent par des lectures à haute voix comme des lectures silencieuses. Elles permettent d'affermir la compréhension des textes complexes [...]. Elles se poursuivent par des échanges et des débats sur les interrogations suscitées et donnent par là l'occasion d'éprouver les libertés et les contraintes de toute interprétation ».

9 L'enfant doit pouvoir " participer à un débat sur l'interprétation d'un texte littéraire en étant capable de vérifier dans le texte ce qui interdit ou permet une interprétation. "Et Virginie Signoret de conclure:« Aujourd'hui, l'interprétation semble jouer un rôle important dans le savoir lire, cette compétence reste malgré tout très liée à celle du dire et comprendre. L'interprétation semble très proche dans les I.O. du concept de compréhension. " Cependant, souligne-t-elle, "ni les programmes, ni les I.O. ne définissent clairement les concepts de compréhension fine et d'interprétation. [...]» comment alors évaluer les progrès de la construction d'une interprétation chez les élèves?

Une deuxième stagiaire, Valérie Margerit, évoquant les I.O. récentes, conclut elle aussi :

"L'interprétation semblerait donc définie ici comme une capacité de compréhension encore plus fine. Il semblerait que la première soit assimilable à comprendre ce qui est écrit et la seconde à faire des hypothèses à partir de ce qui est écrit mais aussi à partir du non-écrit associé à ce que l'enfant sait par ailleurs. Ces compétences d'induction et de déduction seraient donc désormais exigibles dès le cycle III. Mais l'absence totale de définition, de distinction précise entre compréhension et interprétation ne permet pas de cerner cette notion d'interprétation de façon suffisamment précise pour s'appuyer dessus et pouvoir mettre en place une séquence de travail. C'est pourquoi" termine-t-elle "je me suis intéressée aux théories de la lecture et à celles de l'interprétation ».

11 À la question simple que se posent ces jeunes stagiaires : peut-on, au plan didactique, définir véritablement l'interprétation et la différencier de la compréhension? La réponse est non.

Force est d'admettre qu'il faut faire son deuil d'une réponse binaire et accepter d'affronter la complexité de la lecture de la littérature et son développement dès les tout premiers pas en lecture à l'occasion de la rencontre avec les premiers albums en maternelle jusqu'aux oeuvres plus longues du cycle III. On ne trouve pas dans les travaux théoriques de réponse définitive et univoque sur la question des liens entre lire, comprendre, interpréter. On trouve au contraire des points de vue divers selon que les recherches s'intéressent principalement au processus cognitifs du lecteur, selon qu'elles focalisent leur attention sur les textes et les systèmes de significations qu'ils véhiculent et organisent, selon au contraire qu'elles étudient l'activité interprétative du lecteur ou selon que l'on s'intéresse à la diversité des lectures en fonction des contextes ou des communauté d'appartenance du lecteur.

12 Ces multiples recherches qui se sont intéressées à la lecture, renvoient à des champs théoriques très divers: sciences cognitives, herméneutique littéraire, histoire littéraire, socio-critique, anthropologie ou sociologie de la lecture littéraire. On peut renvoyer ici aux principales réponses sur cette question apportées par des auteurs comme Denhières, 
Eco, Leennardt, Jauss, Picard, Rifaterre, Ricœur, Oison. Elles se sont développées principalement entre les années 1975 et 1985 et sont apparues en force dans les recherches didactiques principalement à partir de 1995 avec notamment le colloque international « Pour une lecture littéraire » à Louvain-la-Neuve, sous la direction de J.L. Dufays, L. Gemenne et D. Ledur. Plusieurs autres colloques ont suivi dont les travaux sont régulièrement publiés : Nîmes 1998, Toulouse 1999, Rennes 2000, Grenoble 2002.

Pour le didacticien, le formateur en IUFM, la question est aujourd'hui de comprendre comment se pose et peut se travailler cette question de l'interprétation, comment des modèles didactiques théoriques de la lecture, pour l'instant émergents, la traduisent. Ces modèles en cours d'élaboration sont le fait de la communauté des enseignants chercheurs en didactique du français et de la littérature, mais aussi le fait de pratiques innovantes inventées sur le terrain. Ils ont déjà une histoire dont on peut penser qu'elle a déjà largement contribué à l'arrivée du concept d'interprétation dans les I.O. françaises.

Une autre dimension du travail de «transposition didactique » est de se demander si les recherches et concepts construits par des travaux théoriques pensés à partir de pratiques de lecture d'adultes dont la technique de lecture est fluide, courante au sens premier du terme, peuvent s'appliquer à de jeunes élèves encore mal habiles ou dans un rapport encore très oral à la lecture : le maître, la maman lit, l'enfant peut écouter, rêver, s'échapper, revenir facilement.

J.-C. Chabanne ici même propose notamment de distinguer lecture-déchiffrement et lecture philologique pour identifier des difficultés différentes pouvant empêcher l'entrée dans la lecture compréhension.

15 Autre question importante mais non nouvelle ${ }^{9}$, ces modèles prennent-ils en compte la très grande hétérogénéité culturelle, sociale, langagière des élèves ? Rien n'est moins sûr. Il convient donc de poursuivre les recherches attentives sur ce que disent et font les élèves et leurs maîtres dans leur grande diversité. À noter que la méthodologie de recherche adoptée par M.T. Chemla, M. Dreyfus et M. Cellier (INRP, à paraître) ${ }^{10}$ faisant travailler le même texte dans des classes, des contextes différents, pourrait s'avérer très pertinente pour vérifier ces processus de différenciation.

\section{Essai d'inventaire de quelques tensions nouvelles qui traversent la problématique " lire, comprendre, interpréter »}

16 La lecture des propositions didactiques récemment publiées, les hésitations des stagiaires tentant d'inventer des pratiques répondant aux nouvelles I.O., comme l'observation des pratiques pédagogiques inventées par des professionnels aguerris ayant largement anticipé ces I.O., laissent apparaître un certain nombre de tensions, que fait naître cette question de l'interprétation. Je me contenterai ici de les inventorier. Enseigner c'est toujours en permanence choisir, encore faut-il repérer entre quoi et quoi. Ces tensions nouvelles pourraient faire l'objet d'une réflexion didactique en formation afin de se demander quelles tâches peuvent les objectiver ou les résoudre.

17 Ces tensions mettent l'enseignant face à des dilemmes. Selon les choix qu'ils nécessitent, il adopte consciemment ou pas, diverses postures vis-à-vis de l'élève et du texte littéraire. Dans le meilleur des cas il se fera passeur de textes, de culture, de savoirs, semeur de questions, observateur attentif à l'écoute des réactions et émotions des élèves, empêcheur 
de penser en rond, dans le pire il réduira la lecture de la littérature à un nouveau pensum scolaire.

Les questions qui suivent ne se veulent pas savantes: elles renvoient à des choix pédagogiques à inventer, à essayer; des tâches, des situations de lecture, l'organisation pédagogique du temps consacré à la littérature dans l'emploi du temps officiel de la classe. Elles renvoient à des styles d'enseignement à identifier.

\section{Première tension : l'interprétation précède-t-elle, accompagne-t-elle, ou suit-elle la lecture de l'œuvre?}

Pas de consensus là-dessus. On peut penser que l'horizon d'attente du lecteur, la nature du contrat de lecture négocié avant, les annonces et médiations diverses nécessaires, (On va lire cet album et ensuite on le présentera aux élèves de maternelle), modifie le parcours interprétatif de lecture. On peut aussi imaginer que chemin faisant d'autres itinéraires se feront jour, de même qu'après coup le retour réflexif singulier ou collectif sur la lecture ou l'accrochage ultérieur à d'autres textes (la lecture en réseaux) pourront modifier l'interprétation. À quel niveau et dans quel sens se font ces modifications? la question reste ouverte. C. Tauveron parle d'interprétation N1 pendant, N2 après (Tauveron, 1999, p. 21). On peut penser en suivant A. Jorro (1999), qu'il y a aussi N0 et N + $3+4+5$., très variables selon les textes, leurs thématiques, le degré de familiarité avec le genre, l'auteur, l'illustrateur et que ces mouvements interprétatifs sont très variables aussi selon les lieux de lecture.

\section{Deuxième tension : où situer les limites de l'interprétation à l'école élémentaire ?}

Uniquement dans l'étude du texte c'est-à-dire dans une lecture attentive de la syntaxe, du lexique, du genre, etc? Ou en faisant appel à d'autres éléments culturels, historiques, biographiques, génétiques: $\mathrm{Ah}$, tu aurais pu nous le dire qu'il (Brown, l'auteur, "d'Une histoire à quatre voix »)avait déjà écrit l'histoire de la promenade avant de la présenter sous quatre voix » commentaire des CM1 (mémoire professionnel de Valérie Margerit).

Dans le cas des albums, faut-il travailler conjointement l'interprétation du texte et l'interprétation de l'illustration? Faut-il travailler leur convergence, leur différence? Faut-il focaliser le travail des élèves sur les éléments du texte qui font cohérence ou au contraire les rendre attentifs aux contradictions, aux indéterminations, "à la rhétorique des signes flottants ${ }^{11}$ dont on peut penser qu'elle est un des éléments clé de la littérarité d'un texte et de son pouvoir esthétique. Esthétique qui se donne pour tâche selon Ricœur "l'exploration des manières multiples dont une ceuvre en agissant sur le lecteur, l'affecte... expérience qui combine une passivité et une activité, qui permettent de désigner comme réception du texte l'action même du lire $»^{12}$

La tentation est cependant souvent grande de focaliser l'interprétation sur des aspects inférentiels, pragmatiques pour colmater tous les blancs du texte, cerner le vrai du faux, au risque d'oublier les autres dimensions plus essentielles: esthétiques et éthiques qui questionnent le degré de conformité ou de subversion du texte par rapport à des formes d'écriture (les contes à l'envers), à des stéréotypes (l'image du grand méchant loup), des systèmes de valeurs. Dans Fantastique Maître Renard, par exemple, R. Dahl pose une question métaphorique tout à la fois écologique et politique : les grands fermiers blancs américains ont-ils le droit d'affamer la famille de Renard avec leurs grandes moissonneuses et avec renard la cohorte de tous les petits, tous les laissés pour compte du 
système. Renard et ses amis ont-ils le droit de se révolter, d'aller voler pour vivre ? Quel dilemme ! (le mot est dans le texte). L'interprétation philosophique, qui permet de poser un regard sur le monde est ici plus intéressante qu'une compréhension - interprétation psychologique ou référentielle. Les textes des élèves de CM1 en ZEP ${ }^{13}$ montrent qu'ils en sont fort capables : Khadija écrit :

"Ce que je pense de ce livre:

" Je pense que ce livre est merveilleux parce qu'il y a des aventures et moi j'adore les aventures.

"Mais ce qui m'a plu, c'est que l'auteur a fait des animaux en habits d'êtres humains. Ce qui ne m'a pas plu, c'est quand le renard volait des oies, les canards... parce que d'un côté, c'est bien de voler et d'un côté non ".

Après que le maître lui ait demandé en marge de son texte d'expliquer un peu plus ce qu'elle voulait dire, elle ajoute :« Si on vole pour soi-même c'est pas bien et si Maitre renard vole pour ses enfants, là c'est bien."

Ajoutons pour resituer le contexte que le maitre, par le biais de ce texte littéraire conjugué avec d'autres, tentait à ce moment de faire réfléchir ses élèves sur la question de la loi, dans un contexte de violence politique forte dans une cité marquée par les événements du 11 septembre 2001.

Il arrive aussi que sous le prétexte que les enfants sont de jeunes lecteurs encore mal habiles, les attentes interprétatives du maître se limitent à ne faire travailler l'interprétation que sous sa dimension inférentielle et non pas comme le lieu même d'une pensée esthétique, créative, effervescente. Il suffit de voir des séances de lecture d'albums aux petits pour repérer combien cette deuxième dimension, est non seulement essentielle mais probablement le support de l'instauration d'un goût durable pour la lecture. Très tôt, dès 4,5 ans, les enfants peuvent à condition qu'on les accompagne un peu, comprendre tous les clins d'œil littéraires, humoristiques, axiologiques, linguistiques qui font flores dans la grande saga actuelle des albums sur le loup. Ils ne s'y trompent pas et arrivent à se retrouver très convenablement dans de multiples histoires en abîmes.

\section{Troisième tension : faut-il s'arrêter à des interprétations premières, ou systématiquement chercher à les rendre savantes, distanciées, secondes ?}

Par posture première on entend des postures de lecture du type texte action ${ }^{14}$ (le texte est l'espace fictif d'un investissement psycho affectif fort du sujet: la lecture est très psychologique, très filtrée par des jugement de valeurs plus ou moins moralisateurs, souvent inconscients - le lecteur est lu par le texte dirait Picard). En systématisant au contraire les interprétations plus "élaborées", plus stratégiques, conscientes, ou simplement scolaires (les labels de ces formes scolaires de lectures ne cessent d'évoluer !), on sait qu'on risque de scolariser la lecture littéraire et de la réduire à... des discours sur la lecture. Comment faire alors glisser les élèves d'une posture interprétative à une autre? Comment leur faire construire avec les textes lus des rapports différents?(c'est l'histoire de... En lisant ce texte, j'ai pensé à... je me suis demandé si... À la place du personnage j'aurais... L'auteur organise le suspense en... Après le premier chapitre je pensais que... j'imaginais que... maintenant je me dis que... etc) Chacune de ces amorces ouvre des rapports au texte différents, conduit à des postures interprétatives différentes. Le jeu théâtral, les lectures parodiques peuvent aussi ouvrir à des interprétations ludiques, farfelues, créatives. comment ne pas «manipuler» les élèves par les formes de questionnements? Dans 
"Histoire à quatre voix ", les stagiaires observent que certains élèves peuvent, selon les questions posées, selon l'avancée dans la lecture, selon l'image qu'ils veulent renvoyer d'eux au maître ou au groupe s'investir différemment dans les personnages et se livrer ainsi à des reconfigurations axiologiques fortement symboliques. Le texte fait se rencontrer dans un parc une petite fille de chômeur et un garçonnet des quartiers chics, une chienne de race et un bâtard, un papa déprimé et une maman très collet monté. La même promenade est racontée par quatre voix différentes et laisse les lecteurs à leurs choix interprétatifs tant sur le plan des références culturelles montrées - notamment par l'illustration - que par les formes langagières diverses adoptés par les personnages et transcrites dans des graphies différentes, selon les voix ou les valeurs dessinées mais jamais verbalisées.

\section{Quatrième tension : faut-il ou non favoriser systématiquement l'explicitation, la verbalisation orale ou écrite de l'interprétation?}

L'enjeu est connu: il s'agit de provoquer des conflits d'interprétations, pour obliger l'élève à se décentrer, à dépasser ses premières impressions (les semences disent Terwagne, Vanhulle et Lafontaine ${ }^{15}$. Pourtant ne faut-il pas aussi admettre qu'il est important de laisser une part de l'activité interprétative dans l'ombre ${ }^{16}$ des dialogues secrets avec soi même, avec ses partenaires de pensée privilégiés, avec les héros ou auteurs d'autres livres? Cet espace d'interprétation bien qu'intérieur, conscient, préconscient ou inconscient ne met pas le sujet de la même façon en activité d'interprétation. Les mondes et images qui s'accumulent dans notre inconscient (notre culture : nos outils pour penser le monde et se le représenter) sont le terreau de nos interprétations ultérieures. Il est certain que les verbaliser les modifie, les remet en travail, les classe, les structure, les câble (au sens neuronal) - les théories vygotskiennes du rapport langage - pensée nous le rappellent. Est-ce toujours nécessaire ? La pensée n'at-elle pas besoin de zones incertaines, «molles», «flottantes» (voir supra) pour s'autoriser à inventer, accrocher ensemble des éléments hétéroclites ;

Mais alors quelles tâches astucieuses proposer pour favoriser ces accrochages et tissages culturels entre les œuvres lues? Sont en train d'émerger dans les classes des formes de commentaires nouvelles(À la place du héros tu... Et toi il t'es arrivé de... Toi aussi tu as été victime, témoin de... etc, des formes d'écriture ou de réécritures narratives ou poétiques non académiques, des formes aussi d'interprétation où le jeu théâtral, voire la mise en images, en musique jouent un rôle important). L'objectif commun est de trouver un point d'arrimage : accrocher la culture apportée à quelque chose de connu, de personnel, à un événement, une émotion, une réflexion relevant de l'expérience propre du sujet.

Une autre dérive importante qu'on sent pointer est de faire de la lecture littéraire un simple prétexte pour un travail oral dans la classe.

\section{La dernière tension et non la moindre est de se demander comment didactiser une pratique sociale au demeurant fortement différenciée selon les milieux sociaux}

Car enfin, si interpréter c'est trivialement parler avec l'autre, avoir envie de partager avec lui un point de vue, des impressions devant une tasse de café, entre la poire et le fromage au sujet du dernier film ou du roman à la mode, dans le cercle restreint de ses interlocuteurs habituels, force est d'admettre et les travaux de sociologie de la littérature l'ont abondamment montré que ces pratiques sociales interprétatives sont fort 
différentes d'une catégorie sociale à l'autre. Lesquelles alors mettre en exergue ? faut-il trier ou laisser se construire lentement des cheminements communs et divers à l'intérieur d'une communauté, l'école, qu'il convient de considérer aussi comme un lieu social. On sait par exemple comment les élèves aiment à s'échanger dans la cour des ouvrages qui ne sont pas dans les BCD ou CDI et qu'ils ne souhaitent pas y trouver! Quel rôle d'étayage ou au contraire, d'aveugle non dupe, doit savoir alors jouer le maître?

\section{Conclusion}

Ces questions, on le voit, sont très liées à des conceptions différentes du sujet élève, mais aussi à des points de vue différents sur la lecture littéraire et sa didactique, sur le rôle formateur et éducatif de la culture littéraire. Elles sont largement en débat et nécessitent un travail important et nouveau en formation.

On imagine aisément comment la manière dont seront organisés, dans le temps, l'espace de la classe, les débats interprétatifs, leurs enjeux, la manière dont ils seront ou non précédés par de l'écrit, la nature des textes travaillés, comment tout cela peut modifier considérablement les postures interprétatives des élèves. Le chantier ouvert par les nouvelles I.O. est donc important.

\section{NOTES}

1. Jean Hebrard, Juin 2002, conférence, La Grande Motte.

2. Les travaux sur l'interprétation littéraire se développent fortement entre 1975 et 1985 :

RICEUR : Du texte à l'action. Essai d'herméneutique IIParis, Le Seuil, 1986.

JAUSS : Pour une esthétique de la réception. Paris, Gallimard, 1978 (éd d'origine, 1972).

LEENHARDT et JOZSA : Lire la lecture,le Sycomore, 1982.

RIFATERRE : «l'illusion référentielle » in BARTHES R. et al :Littérature et réalité. Paris, Le Seuil, 1982.

PICARD : La lecture comme jeu.Paris, Minuit, 1986.

ISER W. : L'acte de lecture théorie de l'effet esthétique. Paris, Mardaga, 1985.

ECO U. : Lector in fabula ou la coopération interprétative dans les textes narratifs. Paris, Grasset, 1985 (éd d'origine, 1978).

DUFAYS J.L. : Stéréotypes et lecture.Liège, Mardaga, 1994.

et arrivent dans les I.O. du primaire en 2002 !

3. JORRO A.: thèse de doctoratConstruire du sens en lecture? Opérations dans l'apprentissage d'une stratégie de compréhension de texte par les élèves du cycle III. Université d'Aix-Marseille, 1994.

4. Un certain nombre de notions de narratologie : auteur, narrateur, personnage, héros, point de vue, merveilleux, description, sans compter les sempiternelles schémas quinaire ou actanciel, ont été introduites dans les manuels du primaire sans être véritablement des objets d'étude spécifies par les programmes. Les enseignants du secondaire sont extrêmement surpris lorsqu'ils découvrent qu'elles sont déjà des objets d'étude au primaire. 
5. Il s'agit principalement des travaux de l'équipe INRP dirigée par TAUVERON C. : « comprendre et interpréter les textes à l'école ", in Repères, $\mathrm{N}^{\circ} 19,1999$, et ceux de l'équipe belge : TERWAGNE S., VANHULLE S., LAFONTAINE A. : Les cercles de lecture,Debœck-Duculot, 2001.

6. JORRO A. : Le lecteur interprète.Paris, PUF, 1999, p. 103-118.

7. MARGERIT V. :Une histoire à quatre voix : un essai de séquence d'interprétation littéraire en cycle III. Montpellier, IUFM de Montpellier, 2002.

SIGNORET V. : Une histoire à quatre voix d'Antony Browne : un essai d'interprétation au cycle III. Montpellier, IUFM de Montpellier, 2002.

8. En italique, les citations des I.O.

9. Voir l'article d'Y. REUTER de 1986 : « lire une pratique socio-culturelle », in Pratiques, $\mathrm{N}^{\circ} 52$.

10. Voir aussi la communication de M.T. CHEMLA, colloque « oral », La Grande Motte, juin 2002.

11. BERTIER P. : Le second apprentissage de la lecture.Antrophos, 1999, pp. 70-77.

12. RICCEUR P. : Temps et récit,III. 1985, p. 303.

13. Classe d'Alain Dunas, École Blaise Pascal, Perpignan (série de lectures après le 11 septembre 2001).

14. BUCHETON D. : « les postures du lecteur ", in Lecture privée et lecture scolaire, la question de la littérature à l'école(P. Demougin et J.F. Massol, coord). Grenoble, CRDP Grenoble, 1998.

15. Op. cit.

16. PIBAROT A. : « la lecture privée et le secret », in Lecture privée et lecture scolaire, la question de la littérature à l'école (P. Demougin et J.F. Massol, coord), Grenoble, CRDP Grenoble, 1998.

\section{RÉSUMÉS}

Non disponible

Not Available

\section{AUTEUR}

\section{DOMINIQUE BUCHETON}

IUFM Montpellier, équipe LIRDEF 